\title{
Coupled fixed point results in cone metric spaces for $\tilde{w}$-compatible mappings
}

Hassen Aydi $^{1 *}$, Bessem Samet ${ }^{2}$ and Calogero Vetro ${ }^{3}$

* Correspondence: hassen. aydi@isima.rnu.tn

${ }^{1}$ Institut Supérieur d'Informatique de Mahdia, Université de Monastir, Route de Rjiche, Km 4, BP 35, Mahdia 5121, Tunisie Full list of author information is available at the end of the article

\section{SpringerOpen ${ }^{\circ}$}

\begin{abstract}
In this paper, we introduce the concepts of $\widetilde{w}$-compatible mappings, b-coupled coincidence point and b-common coupled fixed point for mappings $F, G: X \times X \rightarrow$ $X$, where $(X, d)$ is a cone metric space. We establish b-coupled coincidence and bcommon coupled fixed point theorems in such spaces. The presented theorems generalize and extend several well-known comparable results in the literature, in particular the recent results of Abbas et al. [Appl. Math. Comput. 217, 195-202 (2010)]. Some examples are given to illustrate our obtained results. An application to the study of existence of solutions for a system of non-linear integral equations is also considered.
\end{abstract}

2010 Mathematics Subject Classifications: 54H25; 47H10.

Keywords: -compatible mappings, b-coupled coincidence point, b-common coupled fixed point, cone metric space; integral equation

\section{Introduction}

Ordered normed spaces and cones have applications in applied mathematics, for instance, in using Newton's approximation method [1-4] and in optimization theory [5]. K-metric and K-normed spaces were introduced in the mid-20th century ([2]; see also $[3,4,6])$ by using an ordered Banach space instead of the set of real numbers, as the codomain for a metric. Huang and Zhang [7] re-introduced such spaces under the name of cone metric spaces, and went further, defining convergent and Cauchy sequences in the terms of interior points of the underlying cone. Afterwards, many papers about fixed point theory in cone metric spaces were appeared (see, for example, [8-15]).

The following definitions and results will be needed in the sequel.

Definition 1. [4,7]. Let $E$ be a real Banach space. A subset $P$ of $E$ is called a cone if and only if:

(a) $P$ is closed, non-empty and $P \neq\left\{0_{E}\right\}$,

(b) $a, b \in \mathbb{R}, a, b \geq 0, x, y \in P$ imply that $a x+b y \in P$,

(c) $P \cap(-P)=\left\{0_{E}\right\}$,

where $0_{E}$ is the zero vector of $E$.

Given a cone define a partial ordering $\leqslant$ with respect to $P$ by $x \leqslant y$ if and only if $y$ $x \in P$. We shall write $x \ll y$ for $y-x \in \operatorname{Int} P$, where $\operatorname{Int} P$ stands for interior of $P$. Also, we will use $x<y$ to indicate that $x \leqslant y$ and $x \neq y$. The cone $P$ in a normed space (E, $\|\cdot\|)$ is called normal whenever there is a number $k \geq 1$ such that for all $x, y \in E, 0_{E} \preccurlyeq$

(c) 2011 Aydi et al; licensee Springer. This is an Open Access article distributed under the terms of the Creative Commons Attribution License (http://creativecommons.org/licenses/by/2.0), which permits unrestricted use, distribution, and reproduction in any medium, provided the original work is properly cited. 
$x \leqslant y$ implies $\|x\| \leq k\|y\|$. The least positive number satisfying this norm inequality is called the normal constant of $P$.

Definition 2. [7]. Let $X$ be a non-empty set. Suppose that $d: X \times X \rightarrow E$ satisfies:

(d1) $0_{E} \leqslant d(x, y)$ for all $x, y \in X$ and $d(x, y)=0_{E}$ if and only if $x=y$,

(d2) $d(x, y)=d(y, x)$ for all $x, y \in X$,

(d3) $d(x, y) \leqslant d(x, z)+d(z, y)$ for all $x, y, z \in X$.

Then, $d$ is called a cone metric on $X$, and $(X, d)$ is called a cone metric space.

Definition 3. [7]. Let $(X, d)$ be a cone metric space, $\left\{x_{n}\right\}$ a sequence in $X$ and $x \in X$. For every $c \in E$ with $c \gg 0_{E}$, we say that $\left\{x_{n}\right\}$ is

(C1) a Cauchy sequence if there is some $k \in \mathbb{N}$ such that, for all $n, m \geq k, d\left(x_{n}, x_{m}\right)$ $\ll c$,

(C2) a convergent sequence if there is some $k \in \mathbb{N}$ such that, for all $n \geq k, d\left(x_{n}, x\right) \ll$ $c$. Then $x$ is called limit of the sequence $\left\{x_{n}\right\}$.

Note that every convergent sequence in a cone metric space $X$ is a Cauchy sequence. A cone metric space $X$ is said to be complete if every Cauchy sequence in $X$ is convergent in $X$.

Recently, Abbas et al. [8] introduced the concept of $w$-compatible mappings and established coupled coincidence point and coupled point of coincidence theorems for mappings satisfying a contractive condition in cone metric spaces.

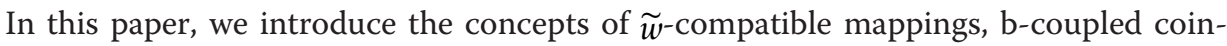
cidence point and b-common coupled fixed point for mappings $F, G: X \times X \rightarrow X$, where $(X, d)$ is a cone metric space. We establish b-coupled coincidence and b-common coupled fixed point theorems in such spaces. The presented theorems generalize and extend several well-known comparable results in the literature, in particular the recent results of Abbas et al. [8] and the result of Olaleru [13]. Some examples and an application to non-linear integral equations are also considered.

\section{Main results}

We start by recalling some definitions.

Definition 4. [16]. An element $(x, y) \in X \times X$ is called a coupled fixed point of mapping $F: X \times X \rightarrow X$ if $x=F(x, y)$ and $y=F(y, x)$.

Definition 5. [17]. An element $(x, y) \in X \times X$ is called

(i) a coupled coincidence point of mappings $F: X \times X \rightarrow X$ and $g: X \rightarrow X$ if $g x=F$ $(x, y)$ and $g y=F(y, x)$, and $(g x, g y)$ is called coupled point of coincidence,

(ii) a common coupled fixed point of mappings $F: X \times X \rightarrow X$ and $g: X \rightarrow X$ if $x$ $=g x=F(x, y)$ and $y=g y=F(y, x)$.

Note that if $g$ is the identity mapping, then Definition 5 reduces to Definition 4 .

Definition 6. [8]. The mappings $F: X \times X \rightarrow X$ and $g: X \rightarrow X$ are called $w$-compatible if $g(F(x, y))=F(g x, g y)$ whenever $g x=F(x, y)$ and $g y=F(y, x)$.

Now, we introduce the following definitions.

Definition 7. An element $(x, y) \in X \times X$ is called

(i) a b-coupled coincidence point of mappings $F, G: X \times X \rightarrow X$ if $G(x, y)=F(x, y)$ and $G(y, x)=F(y, x)$, and $(G(x, y), G(y, x))$ is called b-coupled point of coincidence, 
(ii) a b-common coupled fixed point of mappings $F, G: X \times X \rightarrow X$ if $x=G(x, y)=$ $F(x, y)$ and $y=G(y, x)=F(y, x)$.

Example 1. Let $x=\mathbb{R}$ and $F, G: X \times X \rightarrow X$ the mappings defined by

$$
F(x, y)=(\sin x)(1+y) \text { and } G(x, y)=x^{2}+\left(\frac{\pi}{2}-\frac{2}{\pi}\right) y+1-\frac{\pi^{2}}{4}
$$

for all $x, y \in X$. Then, $(\pi / 2,0)$ is a b-coupled coincidence point of $F$ and $G$, and $(1,0)$ is a b-coupled point of coincidence.

Example 2. Let $X=\mathbb{R}$ and $F, G: X \times X \rightarrow X$ the mappings defined by

$$
F(x, y)=3 x+2 y-6 \text { and } G(x, y)=4 x+3 y-9
$$

for all $x, y \in X$. Then, $(1,2)$ is a b-common coupled fixed point of $F$ and $G$.

Definition 8. The mappings $F, G: X \times X \rightarrow X$ are called $\tilde{w}^{\text {-compatible if }}$

$$
F(G(x, y), G(y, x))=G(F(x, y), F(y, x))
$$

whenever $F(x, y)=G(x, y)$ and $F(y, x)=G(y, x)$.

Example 3. Let $X=\mathbb{R}$ and $F, G: X \times X \rightarrow X$ the mappings defined by

$$
F(x, y)=x^{2}+y^{2} \quad \text { and } \quad G(x, y)=2 x y
$$

for all $x, y \in X$. One can show easily that $(x, y)$ is a b-coupled coincidence point of $F$ and $G$ if and only if $x=y$. Moreover, we have $F(G(x, x), G(x, x))=G(F(x, x), F(x, x))$ for all $x \in X$. Then, $F$ and $G$ are $\tilde{w}$-compatible.

If $(X, d)$ is a cone metric space, we endow the product set $X \times X$ by the cone metric $v$ defined by

$$
v((x, y),(u, v))=d(x, u)+d(y, v), \quad \forall(x, y),(u, v) \in X \times X .
$$

Now, we prove our first result.

Theorem 1. Let $(X, d)$ be a cone metric space with a cone $P$ having non-empty interior. Let $F, G: X \times X \rightarrow X$ be mappings satisfying

(h1) for any $(x, y) \in X \times X$, there exists $(u, v) \in X \times X$ such that $F(x, y)=G(u, v)$ and $F(y, x)=G(v, u)$,

(h2) $\{(G(x, y), G(y, x)): x, y \in X\}$ is a complete subspace of $(X \times X, v)$,

(h3) for any $x, y, u, v \in X$,

$$
\begin{aligned}
& d(F(x, y), F(u, v)) \preccurlyeq a_{1} d(F(x, y), G(x, y))+a_{2} d(F(y, x), G(y, x)) \\
& +a_{3} d(F(u, v), G(u, v))+a_{4} d(F(v, u), G(v, u))+a_{5} d(F(u, v), G(x, y)) \\
& +a_{6} d(F(v, u), G(y, x))+a_{7} d(F(x, y), G(u, v))+a_{8} d(F(y, x), G(v, u)) \\
& +a_{9} d(G(u, v), G(x, y))+a_{10} d(G(v, u), G(y, x)),
\end{aligned}
$$

where $a_{i}, i=1, \ldots, 10$ are nonnegative real numbers such that $\sum_{i=1}^{10} a_{i}<1$. Then $F$ and $G$ have a b-coupled coincidence point $(x, y) \in X \times X$, that is, $F(x, y)=G(x, y)$ and $F(y, x)=G(y, x)$.

Proof. Let $x_{0}$ and $y_{0}$ be two arbitrary points in $X$. By (h1), there exists $\left(x_{1}, y_{1}\right)$ such that

$$
F\left(x_{0}, y_{0}\right)=G\left(x_{1}, y_{1}\right) \text { and } F\left(y_{0}, x_{0}\right)=G\left(y_{1}, x_{1}\right) \text {. }
$$

Continuing this process, we can construct two sequences $\left\{x_{n}\right\}$ and $\left\{y_{n}\right\}$ in $X$ such that

$$
F\left(x_{n}, y_{n}\right)=G\left(x_{n+1}, y_{n+1}\right), \quad F\left(y_{n}, x_{n}\right)=G\left(y_{n+1}, x_{n+1}\right), \quad \forall n \in \mathbb{N} \text {. }
$$


For any $n \in \mathbb{N}$, let $z_{n} \in X$ and $t_{n} \in X$ as follows

$$
z_{n}:=F\left(x_{n}, y_{n}\right)=G\left(x_{n+1}, y_{n+1}\right), \quad t_{n}:=F\left(y_{n}, x_{n}\right)=G\left(y_{n+1}, x_{n+1}\right) .
$$

Now, taking $(x, y)=\left(x_{n}, y_{n}\right)$ and $(u, v)=\left(x_{n+1}, y_{n+1}\right)$ in the considered contractive condition and using (2), we have

$$
\begin{aligned}
& d\left(z_{n}, z_{n+1}\right)=d\left(F\left(x_{n}, y_{n}\right), F\left(x_{n+1}, y_{n+1}\right)\right) \\
& \preccurlyeq a_{1} d\left(F\left(x_{n}, y_{n}\right), G\left(x_{n}, y_{n}\right)\right)+a_{2} d\left(F\left(y_{n}, x_{n}\right), G\left(y_{n}, x_{n}\right)\right) \\
& +a_{3} d\left(F\left(x_{n+1}, y_{n+1}\right), G\left(x_{n+1}, y_{n+1}\right)\right)+a_{4} d\left(F\left(y_{n+1}, x_{n+1}\right), G\left(y_{n+1}, x_{n+1}\right)\right) \\
& +a_{5} d\left(F\left(x_{n+1}, y_{n+1}\right), G\left(x_{n}, y_{n}\right)\right)+a_{6} d\left(F\left(y_{n+1}, x_{n+1}\right), G\left(y_{n}, x_{n}\right)\right) \\
& +a_{7} d\left(F\left(x_{n}, y_{n}\right), G\left(x_{n+1}, y_{n+1}\right)\right)+a_{8} d\left(F\left(y_{n}, x_{n}\right), G\left(y_{n+1}, x_{n+1}\right)\right) \\
& +a_{9} d\left(G\left(x_{n+1}, y_{n+1}\right), G\left(x_{n}, y_{n}\right)\right)+a_{10} d\left(G\left(y_{n+1}, x_{n+1}\right), G\left(y_{n}, x_{n}\right)\right) \\
& =\left(a_{1}+a_{9}\right) d\left(z_{n}, z_{n-1}\right)+\left(a_{2}+a_{10}\right) d\left(t_{n}, t_{n-1}\right)+a_{3} d\left(z_{n+1}, z_{n}\right) \\
& +a_{4} d\left(t_{n+1}, t_{n}\right)+a_{5} d\left(z_{n+1}, z_{n-1}\right)+a_{6} d\left(t_{n+1}, t_{n-1}\right) .
\end{aligned}
$$

Then, using the triangular inequality, one can write for any $n \in \mathbb{N}^{*}$

$$
\begin{aligned}
& \left(1-a_{3}\right) d\left(z_{n}, z_{n+1}\right) \preccurlyeq\left(a_{1}+a_{9}\right) d\left(z_{n}, z_{n-1}\right)+\left(a_{2}+a_{10}\right) d\left(t_{n}, t_{n-1}\right)+a_{4} d\left(t_{n+1}, t_{n}\right) \\
& +a_{5} d\left(z_{n+1}, z_{n}\right)+a_{5} d\left(z_{n}, z_{n-1}\right)+a_{6} d\left(t_{n+1}, t_{n}\right)+a_{6} d\left(t_{n}, t_{n-1}\right) .
\end{aligned}
$$

Therefore,

$$
\begin{aligned}
& \left(1-a_{3}-a_{5}\right) d\left(z_{n}, z_{n+1}\right) \preccurlyeq\left(a_{1}+a_{5}+a_{9}\right) d\left(z_{n}, z_{n-1}\right)+\left(a_{2}+a_{6}+a_{10}\right) d\left(t_{n}, t_{n-1}\right) \\
& +\left(a_{4}+a_{6}\right) d\left(t_{n+1}, t_{n}\right) .
\end{aligned}
$$

Similarly, taking $(x, y)=\left(y_{n}, x_{n}\right)$ and $(u, v)=\left(y_{n+1}, x_{n+1}\right)$ and reasoning as above, we obtain

$$
\begin{aligned}
& \left(1-a_{3}-a_{5}\right) d\left(t_{n}, t_{n+1}\right) \preccurlyeq\left(a_{1}+a_{5}+a_{9}\right) d\left(t_{n}, t_{n-1}\right)+\left(a_{2}+a_{6}+a_{10}\right) d\left(z_{n}, z_{n-1}\right) \\
& +\left(a_{4}+a_{6}\right) d\left(z_{n+1}, z_{n}\right) .
\end{aligned}
$$

Adding (4) to (5), we have

$$
\begin{aligned}
& \left(1-a_{3}-a_{5}\right)\left(d\left(z_{n}, z_{n+1}\right)+d\left(t_{n}, t_{n+1}\right)\right) \preccurlyeq\left(a_{1}+a_{5}+a_{9}\right)\left(\left(d\left(z_{n}, z_{n-1}\right)+d\left(t_{n}, t_{n-1}\right)\right)\right. \\
& +\left(a_{2}+a_{6}+a_{10}\right)\left(d\left(z_{n}, z_{n-1}\right)+d\left(t_{n}, t_{n-1}\right)\right)+\left(a_{4}+a_{6}\right)\left(d\left(z_{n+1}, z_{n}\right)+d\left(t_{n+1}, t_{n}\right)\right) .
\end{aligned}
$$

Let us denote

$$
\delta_{n}=d\left(z_{n}, z_{n+1}\right)+d\left(t_{n}, t_{n+1}\right),
$$

then, we deduce that

$$
\left(1-a_{3}-a_{5}\right) \delta_{n} \preccurlyeq\left(a_{1}+a_{5}+a_{9}+a_{2}+a_{6}+a_{10}\right) \delta_{n-1}+\left(a_{4}+a_{6}\right) \delta_{n} .
$$

On the other hand, we have

$$
\begin{aligned}
& d\left(z_{n+1}, z_{n}\right)=d\left(F\left(x_{n+1}, y_{n+1}\right), F\left(x_{n}, y_{n}\right)\right) \\
& \preccurlyeq a_{1} d\left(F\left(x_{n+1}, y_{n+1}\right), G\left(x_{n+1}, y_{n+1}\right)\right)+a_{2} d\left(F\left(y_{n+1}, x_{n+1}\right), G\left(y_{n+1}, x_{n+1}\right)\right) \\
& +a_{3} d\left(F\left(x_{n}, y_{n}\right), G\left(x_{n}, y_{n}\right)\right)+a_{4} d\left(F\left(y_{n}, x_{n}\right), G\left(y_{n}, x_{n}\right)\right) \\
& +a_{5} d\left(F\left(x_{n}, y_{n}\right), G\left(x_{n+1}, y_{n+1}\right)\right)+a_{6} d\left(F\left(y_{n}, x_{n}\right), G\left(y_{n+1}, x_{n+1}\right)\right) \\
& +a_{7} d\left(F\left(x_{n+1}, y_{n+1}\right), G\left(x_{n}, y_{n}\right)\right)+a_{8} d\left(F\left(y_{n+1}, x_{n+1}\right), G\left(y_{n}, x_{n}\right)\right) \\
& +a_{9} d\left(G\left(x_{n}, y_{n}\right), G\left(x_{n+1}, y_{n+1}\right)\right)+a_{10} d\left(G\left(y_{n}, x_{n}\right), G\left(y_{n+1}, x_{n+1}\right)\right) \\
& =\left(a_{3}+a_{9}\right) d\left(z_{n}, z_{n-1}\right)+\left(a_{4}+a_{10}\right) d\left(t_{n}, t_{n-1}\right)+a_{1} d\left(z_{n+1}, z_{n}\right) \\
& +a_{2} d\left(t_{n+1}, t_{n}\right)+a_{7} d\left(z_{n+1}, z_{n-1}\right)+a_{8} d\left(t_{n+1}, t_{n-1}\right),
\end{aligned}
$$


from which by the triangular inequality, it follows that

$$
\begin{aligned}
& d\left(z_{n+1}, z_{n}\right) \preccurlyeq\left(a_{3}+a_{9}\right) d\left(z_{n}, z_{n-1}\right)+\left(a_{4}+a_{10}\right) d\left(t_{n}, t_{n-1}\right)+a_{1} d\left(z_{n+1}, z_{n}\right) \\
& +a_{2} d\left(t_{n+1}, t_{n}\right)+a_{7} d\left(z_{n+1}, z_{n}\right)+a_{7} d\left(z_{n}, z_{n-1}\right)+a_{8} d\left(t_{n+1}, t_{n}\right)+a_{8} d\left(t_{n}, t_{n-1}\right) .
\end{aligned}
$$

Therefore,

$$
\begin{aligned}
& \left(1-a_{1}-a_{7}\right) d\left(z_{n}, z_{n+1}\right) \preccurlyeq\left(a_{3}+a_{7}+a_{9}\right) d\left(z_{n}, z_{n-1}\right)+\left(a_{4}+a_{8}+a_{10}\right) d\left(t_{n}, t_{n-1}\right) \\
& +\left(a_{2}+a_{8}\right) d\left(t_{n+1}, t_{n}\right) .
\end{aligned}
$$

Similarly, we find

$$
\begin{aligned}
& \left(1-a_{1}-a_{7}\right) d\left(t_{n}, t_{n+1}\right) \preccurlyeq\left(a_{3}+a_{7}+a_{9}\right) d\left(t_{n}, t_{n-1}\right)+\left(a_{4}+a_{8}+a_{10}\right) d\left(z_{n}, z_{n-1}\right) \\
& +\left(a_{2}+a_{8}\right) d\left(z_{n+1}, z_{n}\right) .
\end{aligned}
$$

Summing (8) to (9) and referring to (6), we get

$$
\left(1-a_{1}-a_{7}\right) \delta_{n} \preccurlyeq\left(a_{3}+a_{4}+a_{7}+a_{8}+a_{9}+a_{10}\right) \delta_{n-1}+\left(a_{2}+a_{8}\right) \delta_{n} .
$$

Finally, from (7) and (10), we have for any $n \in \mathbb{N}^{*}$

$$
\left(2-\sum_{i=1}^{8} a_{i}\right) \delta_{n} \preccurlyeq\left(\sum_{i=1}^{10} a_{i}+a_{9}+a_{10}\right) \delta_{n-1},
$$

that is

$$
\delta_{n} \preccurlyeq \alpha \delta_{n-1} \quad \forall n \in \mathbb{N}^{*},
$$

where

$$
\alpha=\frac{\sum_{i=1}^{10} a_{i}+a_{9}+a_{10}}{2-\sum_{i=1}^{8} a_{i}} .
$$

Consequently, we have

$$
0_{E} \preccurlyeq \delta_{n} \preccurlyeq \alpha \delta_{n-1} \preccurlyeq \cdots \preccurlyeq \alpha^{n} \delta_{0} .
$$

If $\delta_{0}=0_{E}$, we get $d\left(z_{0}, z_{1}\right)+d\left(t_{0}, t_{1}\right)=0_{E}$, that is, $z_{0}=z_{1}$ and $t_{0}=t_{1}$. Therefore, from (2) and (6), we have

$$
F\left(x_{0}, y_{0}\right)=G\left(x_{1}, y_{1}\right)=F\left(x_{1}, y_{1}\right)
$$

and

$$
F\left(y_{0}, x_{0}\right)=G\left(y_{1}, x_{1}\right)=F\left(y_{1}, x_{1}\right),
$$

meaning that $\left(x_{1}, y_{1}\right)$ is a b-coupled coincidence point of $F$ and $G$.

Now, assume that $\delta_{0}>0_{E}$. If $m>n$, we have

$$
\begin{gathered}
d\left(z_{m}, z_{n}\right) \preccurlyeq d\left(z_{m}, z_{m-1}\right)+d\left(z_{m-1}, z_{m-2}\right)+\cdots+d\left(z_{n+1}, z_{n}\right), \\
d\left(t_{m}, t_{n}\right) \preccurlyeq d\left(t_{m}, t_{m-1}\right)+d\left(t_{m-1}, t_{m-2}\right)+\cdots+d\left(t_{n+1}, t_{n}\right) .
\end{gathered}
$$

Summing the two above inequalities, we obtain using also (13) and (6)

$$
\begin{aligned}
d\left(z_{m}, z_{n}\right)+d\left(t_{m}, t_{n}\right) & \preccurlyeq \delta_{m-1}+\delta_{m-2}+\cdots+\delta_{n} \\
& \preccurlyeq\left(\alpha^{m-1}+\alpha^{m-1}+\cdots+\alpha^{n}\right) \delta_{0} \\
& \preccurlyeq \frac{\alpha^{n}}{1-\alpha} \delta_{0} .
\end{aligned}
$$


As $0 \leq \sum_{i=1}^{10} a_{i}<1$, we have $0 \leq \alpha<1$. Hence, for any $c \in E$ with $c \gg 0_{E}$, there exists $N \in \mathbb{N}$ such that for any $n \geq N$, we have $\frac{\alpha^{n}}{1-\alpha} \delta_{0} \ll c$. Furthermore, for any $m>n$ $\geq N$, we get

$$
d\left(z_{m}, z_{n}\right)+d\left(t_{m}, t_{n}\right) \ll c .
$$

Thus, we proved that for any $c \gg 0_{E}$, there exists $n \in \mathbb{N}$ such that

$$
v\left(\left(z_{m}, t_{m}\right),\left(z_{n}, t_{n}\right)\right) \ll c, \forall m>n \geq N .
$$

This implies that $\left\{\left(z_{n}, t_{n}\right)\right\}$ is a Cauchy sequence in the cone metric space $(X \times X, v)$. On the other hand, we have $\left(z_{n}, t_{n}\right)=\left(G\left(x_{n+1}, y_{n+1}\right), G\left(y_{n+1}, x_{n+1}\right)\right) \in\{(G(x, y), G(y, x))$ : $x, y \in X\}$ that is a complete subspace of $(X \times X, v)$ (from (h2)). Hence, there exists $(z$, $t) \in\{(G(x, y), G(y, x)): x, y \in X\}$ such that for all $c \gg 0_{E}$, there exists $\mathcal{N} \in \mathbb{N}$ such that

$$
v\left(\left(z_{n}, t_{n}\right),(z, t)\right) \ll c, \forall n \geq \mathcal{N} \text {. }
$$

This implies that there exist $x, y \in X$ such that $z=G(x, y)$ and $t=G(y, x)$ with

$$
z_{n} \rightarrow z=G(x, y) \text { as } n \rightarrow+\infty
$$

and

$$
t_{n} \rightarrow t=G(y, x) \text { as } n \rightarrow+\infty
$$

Now, we prove that $F(x, y)=G(x, y)$ and $F(y, x)=G(y, x)$, that is, $(x, y)$ is a bcoupled coincidence point of $F$ and $G$. First, by the triangular inequality, we have

$$
\begin{aligned}
& d(F(x, y), G(x, y)) \preccurlyeq d\left(F(x, y), F\left(x_{n}, y_{n}\right)\right)+d\left(F\left(x_{n}, y_{n}\right), G(x, y)\right) \\
& =d\left(F(x, y), F\left(x_{n}, y_{n}\right)\right)+d\left(G\left(x_{n+1}, y_{n+1}\right), G(x, y)\right) .
\end{aligned}
$$

On the other hand, applying the contractive condition in (h3), we get

$$
\begin{aligned}
& d\left(F(x, y), F\left(x_{n}, y_{n}\right)\right) \preccurlyeq a_{1} d(F(x, y), G(x, y))+a_{2} d(F(y, x), G(y, x)) \\
& +a_{3} d\left(F\left(x_{n}, y_{n}\right), G\left(x_{n}, y_{n}\right)\right)+a_{4} d\left(F\left(y_{n}, x_{n}\right), G\left(y_{n}, x_{n}\right)\right)+a_{5} d\left(F\left(x_{n}, y_{n}\right), G(x, y)\right) \\
& +a_{6} d\left(F\left(y_{n}, x_{n}\right), G(y, x)\right)+a_{7} d\left(F(x, y), G\left(x_{n}, y_{n}\right)\right)+a_{8} d\left(F(y, x), G\left(y_{n}, x_{n}\right)\right) \\
& +a_{9} d\left(G\left(x_{n}, y_{n}\right), G(x, y)\right)+a_{10} d\left(G\left(y_{n}, x_{n}\right), G(y, x)\right) \\
& =a_{1} d(F(x, y), G(x, y))+a_{2} d(F(y, x), G(y, x))+a_{3} d\left(z_{n}, z_{n-1}\right)+a_{4} d\left(t_{n}, t_{n-1}\right) \\
& +a_{5} d\left(z_{n}, G(x, y)\right)+a_{6} d\left(t_{n}, G(y, x)\right)+a_{7} d\left(F(x, y), z_{n-1}\right)+a_{8} d\left(F(y, x), t_{n-1}\right) \\
& +a_{9} d\left(z_{n-1}, G(x, y)\right)+a_{10} d\left(t_{n-1}, G(y, x)\right) .
\end{aligned}
$$

Combining the above inequality with (16), and using again the triangular inequality, we get

$$
\begin{aligned}
& d(F(x, y), G(x, y)) \preccurlyeq a_{1} d(F(x, y), G(x, y))+a_{2} d(F(y, x), G(y, x))+a_{3} d\left(z_{n}, z_{n-1}\right) \\
& +a_{4} d\left(t_{n}, t_{n-1}\right)+a_{5} d\left(z_{n}, G(x, y)\right)+a_{6} d\left(t_{n}, G(y, x)\right)+a_{7} d(F(x, y), G(x, y)) \\
& +a_{7} d\left(G(x, y), z_{n-1}\right)+a_{8} d(F(y, x), G(y, x))+a_{8} d\left(G(y, x), t_{n-1}\right) \\
& +a_{9} d\left(z_{n-1}, G(x, y)\right)+a_{10} d\left(t_{n-1}, G(y, x)\right)+d\left(G\left(x_{n+1}, y_{n+1}\right), G(x, y)\right) .
\end{aligned}
$$

Therefore, we have

$$
\begin{aligned}
& \left(1-a_{1}-a_{7}\right) d(F(x, y), G(x, y))-\left(a_{2}+a_{8}\right) d(F(y, x), G(y, x)) \\
& \preccurlyeq a_{3} d\left(z_{n}, z_{n-1}\right)+a_{4} d\left(t_{n}, t_{n-1}\right)+\left(a_{5}+1\right) d\left(z_{n}, G(x, y)\right)+a_{6} d\left(t_{n}, G(y, x)\right) \\
& +\left(a_{7}+a_{9}\right) d\left(G(x, y), z_{n-1}\right)+\left(a_{8}+a_{10}\right) d\left(G(y, x), t_{n-1}\right) .
\end{aligned}
$$


Similarly, one can find

$$
\begin{aligned}
& \left(1-a_{1}-a_{7}\right) d(F(y, x), G(y, x))-\left(a_{2}+a_{8}\right) d(F(x, y), G(x, y)) \\
& \preccurlyeq a_{3} d\left(t_{n}, t_{n-1}\right)+a_{4} d\left(z_{n}, z_{n-1}\right)+\left(a_{5}+1\right) d\left(t_{n}, G(y, x)\right)+a_{6} d\left(z_{n}, G(x, y)\right) \\
& +\left(a_{7}+a_{9}\right) d\left(G(y, x), t_{n-1}\right)+\left(a_{8}+a_{10}\right) d\left(G(x, y), z_{n-1}\right) .
\end{aligned}
$$

Summing (17) and (18), we get

$$
\begin{aligned}
& \left(1-a_{1}-a_{2}-a_{7}-a_{8}\right)(d(F(x, y), G(x, y))+d(F(y, x), G(y, x))) \\
& \preccurlyeq\left(a_{3}+a_{4}\right) \delta_{n-1}+\left(a_{5}+a_{6}+1\right)\left(d\left(z_{n}, G(x, y)\right)+d\left(t_{n}, G(y, x)\right)\right) \\
& +\left(a_{7}+a_{8}+a_{9}+a_{10}\right)\left(d\left(G(y, x), t_{n-1}\right)+d\left(G(x, y), z_{n-1}\right)\right) \\
& \preccurlyeq \delta_{n-1}+2 d\left(z_{n}, G(x, y)\right)+2 d\left(t_{n}, G(y, x)\right)+d\left(G(y, x), t_{n-1}\right)+d\left(G(x, y), z_{n-1}\right) .
\end{aligned}
$$

Therefore, we have

$$
\begin{aligned}
& d(F(x, y), G(x, y))+d(F(y, x), G(y, x)) \preccurlyeq \alpha \delta_{n-1}+\beta d\left(z_{n}, G(x, y)\right) \\
& +\gamma d\left(t_{n}, G(y, x)\right)+\theta d\left(G(y, x), t_{n-1}\right)+\varrho d\left(G(x, y), z_{n-1}\right),
\end{aligned}
$$

where

$$
\alpha=\theta=\varrho=\frac{1}{1-a_{1}-a_{2}-a_{7}-a_{8}}, \beta=\gamma=\frac{2}{1-a_{1}-a_{2}-a_{7}-a_{8}} .
$$

From (13), (14) and (15), for any $c \gg 0_{E}$, there exists $N \in \mathbb{N}$ such that

$$
\delta_{n-1} \preccurlyeq \frac{c}{5 \alpha}, d\left(z_{n}, G(x, y)\right) \preccurlyeq \frac{c}{5 \max \{\beta, \varrho\}}, d\left(t_{n}, G(\gamma, x)\right) \preccurlyeq \frac{c}{5 \max \{\gamma, \theta\}},
$$

for all $n \geq N$. Thus, for all $n \geq N$, we have

$$
d(F(x, y), G(x, y))+d(F(y, x), G(y, x)) \preccurlyeq \frac{c}{5}+\frac{c}{5}+\frac{c}{5}+\frac{c}{5}+\frac{c}{5}=c .
$$

It follows that $d(F(x, y), G(x, y))=d(F(y, x), G(y, x))=0_{E}$, that is, $F(x, y)=G(x, y)$ and $F(y, x)=G(y, x)$. Then, we proved that $(x, y)$ is a b-coupled coincidence point of the mappings $F$ and $G$.

As consequences of Theorem 1, we give the following corollaries.

Corollary 1 . Let $(X, d)$ be a cone metric space with a cone $P$ having non-empty interior. Let $F, G: X \times X \rightarrow X$ be mappings satisfying

(h1) for any $(x, y) \in X \times X$, there exists $(u, v) \in X \times X$ such that $F(x, y)=G(u, v)$ and $F(y, x)=G(v, u)$,

(h2) $\{(G(x, y), G(y, x)): x, y \in X\}$ is a complete subspace of $(X \times X, v)$,

(h3) for any $x, y, u, v \in X$,

$$
\begin{aligned}
& d(F(x, y), F(u, v)) \preccurlyeq \alpha_{1}(d(F(x, y), G(x, y))+d(F(y, x), G(y, x))) \\
& +\alpha_{2}(d(F(u, v), G(u, v))+d(F(v, u), G(v, u)))+\alpha_{3}(d(F(u, v), G(x, y)) \\
& +d(F(v, u), G(y, x)))+\alpha_{4}(d(F(x, y), G(u, v))+d(F(y, x), G(v, u))) \\
& +\alpha_{5}(d(G(u, v), G(x, y))+d(G(v, u), G(y, x))),
\end{aligned}
$$

where $\alpha_{i}, i=1, \ldots, 5$ are nonnegative real numbers such that $\sum_{i=1}^{5} \alpha_{i}<1 / 2$. Then $F$ and $G$ have a b-coupled coincidence point $(x, y) \in X \times X$, that is, $F(x, y)=G(x, y)$ and $F(y, x)=G(y, x)$.

Corollary 2 . Let $(X, d)$ be a cone metric space with a cone $P$ having non-empty interior, $F: X \times X \rightarrow X$ and $g: X \rightarrow X$ be mappings satisfying 


$$
\begin{aligned}
& d(F(x, y), F(u, v)) \preccurlyeq a_{1} d(F(x, y), g x)+a_{2} d(F(y, x), g y)+a_{3} d(F(u, v), g u) \\
& +a_{4} d(F(v, u), g v)+a_{5} d(F(u, v), g x)+a_{6} d(F(v, u), g y)+a_{7} d(F(x, y), g u) \\
& +a_{8} d(F(y, x), g v)+a_{9} d(g u, g x)+a_{10} d(g v, g y),
\end{aligned}
$$

for all $x, y, u, v \in X$, where $a_{i}, i=1, \ldots, 10$ are nonnegative real numbers such that $\sum_{i=1}^{10} a_{i}<1$. If $F(X \times X) \subseteq g(X)$ and $g(X)$ is a complete subset of $X$, then $F$ and $g$ have a coupled coincidence point in $X$, that is, there exists $(x, y) \in X \times X$ such that $g x=F(x$, $y$ ) and $g y=F(y, x)$.

Proof. Consider the mapping $G: X \times X \rightarrow X$ defined by

$$
G(x, y)=g x, \quad \forall x, y \in X .
$$

We will check that all the hypotheses of Theorem 1 are satisfied.

- Hypothesis (h1):

Let $(x, y) \in X \times X$. Since $F(X \times X) \subseteq g(X)$, there exists $u \in X$ such that $F(x, y)=g u=$ $G(u, v)$ for any $v \in X$. Then, (h1) is satisfied.

- Hypothesis (h2):

Let $\left\{x_{n}\right\}$ and $\left\{y_{n}\right\}$ be two sequences in $X$ such that $\left\{\left(G\left(x_{n}, y_{n}\right), G\left(y_{n}, x_{n}\right)\right)\right\}$ is a Cauchy sequence in $(X \times X, v)$. Then, for every $c \gg 0_{E}$, there exists $N \in \mathbb{N}$ such that

$$
v\left(\left(G\left(x_{n}, y_{n}\right), G\left(y_{n}, x_{n}\right)\right),\left(G\left(x_{m}, y_{m}\right), G\left(y_{m}, x_{m}\right)\right)\right) \ll c, \forall n, m \geq N,
$$

that is,

$$
d\left(g x_{n}, g x_{m}\right)+d\left(g y_{n}, g y_{m}\right) \ll c, \quad \forall n, m \geq N .
$$

This implies that $\left\{g x_{n}\right\}$ and $\left\{g y_{n}\right\}$ are Cauchy sequences in $(g(X), d)$. Since $g(X)$ is complete, there exist $x, y \in X$ such that

$$
g x_{n} \rightarrow g x \text { and } g y_{n} \rightarrow g y^{\prime}
$$

that is,

$$
G\left(x_{n}, y_{n}\right) \rightarrow G(x, y) \quad \text { and } \quad G\left(y_{n}, x_{n}\right) \rightarrow G(y, x) .
$$

Therefore,

$$
\left(G\left(x_{n}, y_{n}\right), G\left(y_{n}, x_{n}\right)\right) \rightarrow(G(x, y), G(y, x)) \text { in }(X \times X, v) .
$$

Then, $\{(G(x, y), G(y, x)): x, y \in X\}$ is a complete subspace of $(X \times X, v)$, and so the hypothesis (h2) is satisfied.

- Hypothesis (h3):

The hypothesis (h3) follows immediately from (19).

Now, all the hypotheses of Theorem 1 are satisfied. Then, $F$ and $G$ have a b-coupled coincidence point $(x, y) \in X \times X$, that is, $F(x, y)=G(x, y)=g x$ and $F(y, x)=G(y, x)=$ gy. Thus, $(x, y)$ is a coupled coincidence point of $F$ and $g$

Corollary 3. Let $(X, d)$ be a cone metric space with a cone $P$ having non-empty interior, $F: X \times X \rightarrow X$ and $g: X \rightarrow X$ be mappings satisfying

$$
\begin{aligned}
& d(F(x, y), F(u, v)) \preccurlyeq \alpha_{1}(d(F(x, y), g x)+d(g u, g x))+\alpha_{2}(d(F(y, x), g y) \\
& +d(F(v, u), g v))+\alpha_{3}(d(F(u, v), g x)+d(F(x, y), g u))+\alpha_{4}(d(F(v, u), g y) \\
& +d(F(y, x), g v))+\alpha_{5}(d(F(u, v), g u)+d(g v, g y)),
\end{aligned}
$$


for all $x, y, u, v \in X$, where $\alpha_{i}, i=1, \ldots, 5$ are nonnegative real numbers such that $\sum_{i=1}^{5} \alpha_{i}<1 / 2$. If $F(X \times X) \subseteq g(X)$ and $g(X)$ is a complete subset of $X$, then $F$ and $g$ have a coupled coincidence point in $X$, that is, there exists $(x, y) \in X \times X$ such that $g x$ $=F(x, y)$ and $g y=F(y, x)$.

\section{Remark 1.}

- Putting $a_{2}=a_{4}=a_{6}=a_{8}=0$ in Corollary 2, we obtain Theorem 2.4 of Abbas et al. [8];

- Putting $\alpha_{2}=\alpha_{4}=0$ in Corollary 3, we obtain Corollary 2.5 of [8].

Now, we are ready to state and prove a result of b-common coupled fixed point.

Theorem 2. Let $F, G: X \times X \rightarrow X$ be two mappings which satisfy all the conditions of Theorem 1. If $F$ and $G$ are $\tilde{w}$-compatible, then $F$ and $G$ have a unique b-common coupled fixed point. Moreover, the b-common coupled fixed point of $F$ and $G$ is of the form $(u, u)$ for some $u \in X$.

Proof. First, we'll show that the b-coupled point of coincidence is unique. Suppose that $(x, y)$ and $\left(x^{*}, y^{*}\right) \in X \times X$ with $G(x, y)=F(x, y), G(y, x)=F(y, x), F\left(x^{*}, y^{*}\right)=G\left(x^{*}\right.$, $\left.y^{*}\right)$ and $F\left(y^{*}, x^{*}\right)=G\left(y^{*}, x^{*}\right)$. Using (h3), we get

$$
\begin{aligned}
& d\left(G(x, y), G\left(x^{*}, y^{*}\right)\right)=d\left(F(x, y), F\left(x^{*}, y^{*}\right)\right) \\
& \preccurlyeq a_{1} d(F(x, y), G(x, y))+a_{2} d(F(y, x), G(y, x))+a_{3} d\left(F\left(x^{*}, y^{*}\right), G\left(x^{*}, y^{*}\right)\right) \\
& +a_{4} d\left(F\left(y^{*}, x^{*}\right), G\left(y^{*}, x^{*}\right)\right)+a_{5} d\left(F\left(x^{*}, y^{*}\right), G(x, y)\right)+a_{6} d\left(F\left(y^{*}, x^{*}\right), G(y, x)\right) \\
& +a_{7} d\left(F(x, y), G\left(x^{*}, y^{*}\right)\right)+a_{8} d\left(F(y, x), G\left(y^{*}, x^{*}\right)\right)+a_{9} d\left(G\left(x^{*}, y^{*}\right), G(x, y)\right) \\
& +a_{10} d\left(G\left(y^{*}, x^{*}\right), G(y, x)\right) \\
& =\left(a_{5}+a_{7}+a_{9}\right) d\left(G(x, y), G\left(x^{*}, y^{*}\right)\right)+\left(a_{6}+a_{8}+a_{10}\right) d\left(G(y, x), G\left(y^{*}, x^{*}\right)\right) .
\end{aligned}
$$

Similarly, we obtain

$$
\begin{aligned}
& d\left(G(y, x), G\left(y^{*}, x^{*}\right)\right) \preccurlyeq\left(a_{5}+a_{7}+a_{9}\right) d\left(G(y, x), G\left(y^{*}, x^{*}\right)\right) \\
& +\left(a_{6}+a_{8}+a_{10}\right) d\left(G(x, y), G\left(x^{*}, y^{*}\right)\right) .
\end{aligned}
$$

Therefore, summing the two previous inequalities, we get

$$
\begin{aligned}
& d\left(G(x, y), G\left(x^{*}, y^{*}\right)\right)+d\left(G(y, x), G\left(y^{*}, x^{*}\right)\right) \\
& \preccurlyeq\left(a_{5}+a_{6}+a_{7}+a_{8}+a_{9}+a_{10}\right)\left(d\left(G(y, x), G\left(y^{*}, x^{*}\right)\right)+d\left(G(x, y), G\left(x^{*}, y^{*}\right)\right)\right) .
\end{aligned}
$$

Since $a_{5}+a_{6}+a_{7}+a_{8}+a_{9}+a_{10}<1$, we obtain

$$
d\left(G(x, y), G\left(x^{*}, y^{*}\right)\right)+d\left(G(y, x), G\left(y^{*}, x^{*}\right)\right)=0_{E},
$$

which implies that

$$
G(x, y)=G\left(x^{*}, y^{*}\right), \quad G(y, x)=G\left(y^{*}, x^{*}\right),
$$

meaning the uniqueness of the b-coupled point of coincidence of $F$ and $G$, that is, ( $G$ $(x, y), G(y, x))$. 
Again, using (h3), we have

$$
\begin{aligned}
& d\left(G(x, y), G\left(y^{*}, x^{*}\right)\right)=d\left(F(x, y), F\left(y^{*}, x^{*}\right)\right) \\
& \preccurlyeq a_{1} d(F(x, y), G(x, y))+a_{2} d(F(y, x), G(y, x))+a_{3} d\left(F\left(y^{*}, x^{*}\right), G\left(y^{*}, x^{*}\right)\right) \\
& +a_{4} d\left(F\left(x^{*}, y^{*}\right), G\left(x^{*}, y^{*}\right)\right)+a_{5} d\left(F\left(y^{*}, x^{*}\right), G(x, y)\right)+a_{6} d\left(F\left(x^{*}, y^{*}\right), G(y, x)\right) \\
& +a_{7} d\left(F(x, y), G\left(y^{*}, x^{*}\right)\right)+a_{8} d\left(F(y, x), G\left(x^{*}, y^{*}\right)\right)+a_{9} d\left(G\left(y^{*}, x^{*}\right), G(x, y)\right) \\
& +a_{10} d\left(G\left(x^{*}, y^{*}\right), G(y, x)\right) \\
& =\left(a_{5}+a_{7}+a_{9}\right) d\left(G(x, y), G\left(y^{*}, x^{*}\right)\right)+\left(a_{6}+a_{8}+a_{10}\right) d\left(G(y, x), G\left(x^{*}, y^{*}\right)\right) .
\end{aligned}
$$

Similarly,

$$
\begin{aligned}
& d\left(G(y, x), G\left(x^{*}, y^{*}\right)\right) \preccurlyeq\left(a_{5}+a_{7}+a_{9}\right) d\left(G(y, x), G\left(x^{*}, y^{*}\right)\right) \\
& +\left(a_{6}+a_{8}+a_{10}\right) d\left(G(x, y), G\left(y^{*}, x^{*}\right)\right) .
\end{aligned}
$$

A summation gives

$$
\begin{aligned}
& d\left(G(x, y), G\left(y^{*}, x^{*}\right)\right)+d\left(G(y, x), G\left(x^{*}, y^{*}\right)\right) \\
& \preccurlyeq\left(a_{5}+a_{6}+a_{7}+a_{8}+a_{9}+a_{10}\right)\left(d\left(G(y, x), G\left(x^{*}, y^{*}\right)\right)+d\left(G(x, y), G\left(y^{*}, x^{*}\right)\right)\right) .
\end{aligned}
$$

The fact that $a_{5}+a_{6}+a_{7}+a_{8}+a_{9}+a_{10}<1$ yields that

$$
G(x, y)=G\left(y^{*}, x^{*}\right), \quad G(y, x)=G\left(x^{*}, y^{*}\right) .
$$

In view of (20) and (21), one can assert that

$$
G(x, y)=G(y, x) .
$$

This means that the unique b-coupled point of coincidence of $F$ and $G$ is $(G(x, y), G$ $(x, y))$.

Now, let $u=G(x, y)$, then we have $u=G(x, y)=F(x, y)=G(y, x)=F(y, x)$. Since $F$ and $G$ are $\tilde{w}^{\text {-compatible, we have }}$

$$
F(G(x, y), G(y, x))=G(F(x, y), F(y, x)),
$$

that is, thanks to $(22)$

$$
\begin{aligned}
F(u, u) & =F(G(x, y), G(x, y))=F(G(x, y), G(y, x))=G(F(x, y), F(y, x)) \\
& =G(G(x, y), G(y, x))=G(G(x, y), G(x, y)) \\
& =G(u, u) .
\end{aligned}
$$

Consequently, $(u, u)$ is a b-coupled coincidence point of $F$ and $G$, and so $(G(u, u), G$ $(u, u))$ is a b-coupled point of coincidence of $F$ and $G$, and by its uniqueness, we get $G$ $(u, u)=G(x, y)$. Thus, we obtain

$$
u=G(x, y)=G(u, u)=F(u, u) .
$$

Hence, $(u, u)$ is the unique b-common coupled fixed point of $F$ and $G$. This makes end to the proof.

Corollary 4. Let $F: X \times X \rightarrow X$ and $g: X \rightarrow X$ be two mappings which satisfy all the conditions of Corollary 2. If $F$ and $g$ are $w$-compatible, then $F$ and $g$ have a unique common coupled fixed point. Moreover, the common fixed point of $F$ and $g$ is of the form $(u, u)$ for some $u \in X$.

Proof. From the proof of Corollary 2 and the result given by Theorem 2, we have

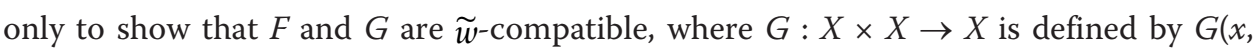


$y)=g x$ for all $x, y \in X$. Let $(x, y) \in X \times X$ such that $F(x, y)=G(x, y)$ and $F(y, x)=G(y$, $x$ ). From the definition of $G$, we get $F(x, y)=g x$ and $F(y, x)=g y$. Since $F$ and $g$ are $w$ compatible, this implies that

$$
g(F(x, y))=F(g x, g y) .
$$

Using (23), we have

$$
F(G(x, y), G(y, x))=F(g x, g y)=g(F(x, y))=G(F(x, y), F(y, x)) .
$$

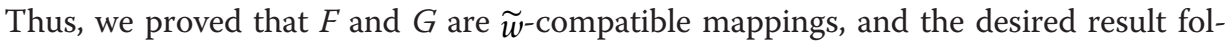
lows immediately from Theorem 2.

Remark 2. Corollary 4 generalizes Theorem 2.11 of [8].

Corollary 5. [13]. Let $(X, d)$ be a cone metric space and $f, g: X \rightarrow X$ be mappings such that

$$
\begin{gathered}
d(f x, f u) \preccurlyeq a_{1} d(f x, g x)+a_{2} d(f u, g u)+a_{3} d(f u, g x) \\
+a_{4} d(f x, g u)+a_{5} d(g u, g x)
\end{gathered}
$$

for all $x, u \in X$, where $\alpha_{i} \in[0,1), i=1, \ldots, 5$ and $\sum_{i=1}^{5} \alpha_{i}<1$. Suppose that $f$ and $g$ are weakly compatible, $f(X) \subseteq g(X)$ and $g(X)$ is a complete subspace of $X$. Then the mappings $f$ and $g$ have a unique common fixed point.

Proof. Consider the mappings $F, G: X \times X \rightarrow X$ defined by $F(x, y)=f x$ and $G(x, y)=$ $g x$ for all $x, y \in X$. Then, the contractive condition (24) implies that

$$
\begin{gathered}
d(F(x, y), F(u, v)) \preccurlyeq a_{1} d(F(x, y), G(x, y))+a_{2} d(F(u, v), G(u, v)) \\
+a_{3} d(F(u, v), G(x, y))+a_{4} d(F(x, y), G(u, v))+a_{5} d(G(u, v), G(x, y)) .
\end{gathered}
$$

Then, $F$ and $G$ satisfy the hypothesis ( $h 3$ ) of Theorem 1 . Clearly, hypothesis $(h 1)$ of Theorem 1 is satisfied since $f(X) \subseteq g(X)$. The hypothesis (h2) is also satisfied since $g(X)$ is a complete subspace of $X$.

Now, we will show that $F$ and $G$ are $\tilde{w}_{\text {-compatible mappings. Let }(x, y) \in X \times X \text { such }}$ that $F(x, y)=G(x, y)$ and $F(y, x)=G(y, x)$. This implies that $f x=g x$. Since $f$ and $g$ are weakly compatible, we have $f(g x)=g(f x)$. Then, we have

$$
F(G(x, y), G(y, x))=F(g x, g y)=f(g x)=g(f x)=g(F(x, y))=G(F(x, y), F(y, x)) .
$$

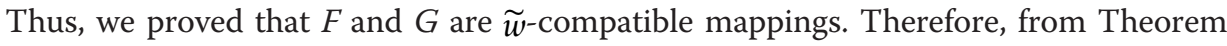
2, $F$ and $G$ have a unique b-common coupled fixed point $(u, u) \in X \times X$ such that $u=$ $F(u, u)=G(u, u)$, that is, $u=f u=g u$. This makes end to the proof.

Now, we give an example to illustrate our obtained results.

Example 4. Let $X=[0,1]$ endowed with the standard metric $d(x, y)=|x-y|$ for all $x, y \in X$. Define the mappings $G, F: X \times X \rightarrow X$ by

$$
G(x, y)=\left\{\begin{array}{ll}
x-y & \text { if } x \geq y \\
0 & \text { if } x<y
\end{array} \text { and } F(x, y)=\left\{\begin{array}{ll}
\frac{x-y}{3} & \text { if } x \geq y . \\
0 & \text { if } x<y
\end{array}\right. \text {. }\right.
$$

We will check that all the hypotheses of Theorem 1 are satisfied.

- Hypothesis (h1): 
Let us prove that for any $x, y \in X$, there exist $u, v \in X$ such that

$$
\left\{\begin{array}{l}
F(x, y)=G(u, v) \\
F(y, x)=G(v, u)
\end{array}\right.
$$

Let $(x, y) \in X \times X$ be fixed. We consider the following cases.

Case-1: $x=y$.

In this case, $F(x, y)=0=G(x, y)$ and $F(y, x)=0=G(y, x)$.

Case-2: $x>y$.

In this case, we have

$$
F(x, y)=\frac{x-y}{3}=G(x / 3, y / 3) \text { and } F(y, x)=0=G(y / 3, x / 3) .
$$

Case-3: $x<y$.

In this case, we have

$$
F(x, y)=0=G(x / 3, y / 3) \text { and } F(y, x)=\frac{y-x}{3}=G(y / 3, x / 3) .
$$

Thus, we proved that ( $h 1)$ is satisfied.

- Hypothesis (h2):

Let us prove that $\Lambda:=\{(G(x, y), G(y, x)): x, y \in[0,1]\}$ is a complete subspace of $([0$, $1] \times[0,1], v)$. Define the function $\phi:[0,1] \times[0,1] \rightarrow \mathbb{R}^{2}$ by

$$
\varphi(x, y)=(G(x, y), G(y, x)) \text { for all } x, y \in[0,1] .
$$

Since $\phi$ is continuous and $[0,1]$ is compact, then $\Lambda=\phi([0,1] \times[0,1])$ is compact. On the other hand, $([0,1] \times[0,1], v)$ is complete. Then, we deduce that $\Lambda$ is complete.

- Hypothesis (h3):

For all $x, y, u, v \in X$, we have

$$
\begin{aligned}
d(F(x, y), F(u, v)) & =|F(x, y)-F(u, v)| \\
& \leq \frac{1}{3}|G(x, y)-G(u, v)| \\
& =\frac{1}{3} d(G(x, y), G(u, v)) .
\end{aligned}
$$

Then, (h3) is satisfied with $a_{1}=a_{2}=\ldots=a_{8}=a_{10}=0$ and $a_{9}=1 / 3$.

All the required hypotheses of Theorem 1 are satisfied. Consequently, $F$ and $G$ have $a$ b-coupled coincidence point.

In this case, for any $x, y \in[0,1],(x, y)$ is a b-coupled coincidence point if and only if $x=y$. Moreover, we have

$$
F(G(x, x), G(x, x))=F(0,0)=0=G(0,0)=G(F(x, x), F(x, x)) .
$$

This implies that $F$ and $G$ are $\widetilde{w}$-compatible. Applying our Theorem 2, we obtain the existence and uniqueness of b-common coupled fixed point of $F$ and $G$. In this example, $(0,0)$ is the unique b-common coupled fixed point.

\section{Application}

In this section, we study the existence of solutions of a system of nonlinear integral equations using the results proved in Section 2. 
Consider the following system of integral equations:

$$
\begin{aligned}
& F(x, y)(t)=\int_{0}^{T} k(t, s) f(s, x(s), y(s)) d s+a(t), \\
& F(y, x)(t)=\int_{0}^{T} k(t, s) f(s, y(s), x(s)) d s+a(t),
\end{aligned}
$$

where $t \in[0, T], T>0$.

Let $X=C([0, T], \mathbb{R})$ be the set of continuous functions defined on $[0, T]$ endowed with the metric given by

$$
d(u, v)=\sup _{t \in[0, T]}|u(t)-v(t)| \text { for all } u, v \in X .
$$

We consider the following assumptions:
(a) $k:[0, T] \times[0, T] \rightarrow \mathbb{R}$ is a continuous function,
(b) $a \in C([0, T], \mathbb{R})$,
(c) $f:[0, T] \times \mathbb{R} \times \mathbb{R} \rightarrow \mathbb{R}$ is a continuous function,
(d) $G: C([0, T], \mathbb{R}) \times C([0, T], \mathbb{R}) \rightarrow C([0, T], \mathbb{R})$ is a mapping satisfying:
(d.1) For all $x, y \in C([0, T], \mathbb{R})$, there exist $u, v \in C([0, T], \mathbb{R})$ such that

$$
\begin{aligned}
& G(u, v)(t)=\int_{0}^{T} k(t, s) f(s, x(s), y(s)) d s+a(t), \\
& G(v, u)(t)=\int_{0}^{T} k(t, s) f(s, y(s), x(s)) d s+a(t),
\end{aligned}
$$

for all $t \in[0, T]$,

(d.2) The set $\{(G(x, y), G(y, x)): x, y \in C([0, T], \mathbb{R})\}$ is closed,

(e) For all $t \in[0, T]$, for all $x, y, u, v \in X$, we have

$$
|f(t, x(t), y(t))-f(t, u(t), v(t))| \leq|G(x, y)(t)-G(u, v)(t)|,
$$

(f) $\sup _{s, t \in I}|k(t, s)|=M<1 / T$.

Now, we formulate our result.

Theorem 3. Under hypotheses (a) - (f), system (25)-(26) has at least one solution in $C$ $([0, T], \mathbb{R})$.

Proof. We consider the operator $F: X \times X \rightarrow X$ defined by

$$
F(x, y)(t)=\int_{0}^{T} k(t, s) f(s, x(s), y(s)) d s+a(t), \quad t \in[0, T] .
$$

It is easy to show that $(x, y)$ is a solution to (25)-(26) if and only if $(x, y)$ is a bcoupled coincidence point of $F$ and $G$. To establish the existence of such a point, we will use our Theorem 1 . Then, we have to check that all the hypotheses of Theorem 1 are satisfied.

- Hypotheses (h1)-(h2) follow immediately from assumption (d).

- Hypothesis (h3): Let $x, y, u, v \in X$. For all $t \in[0, T]$, we have

$$
|F(x, y)(t)-F(u, v)(t)| \leq \int_{0}^{T}|k(t, s)||f(t, x(s), y(s))-f(t, u(s), v(s))| d s .
$$


Using condition (e), we get

$$
\begin{aligned}
|F(x, y)(t)-F(u, v)(t)| & \leq \int_{0}^{T}|k(t, s)||G(x, y)(s)-G(u, v)(s)| d s \\
& \leq\left(\int_{0}^{T}|k(t, s)| d s\right) d(G(x, y), G(u, v)) .
\end{aligned}
$$

Using condition (f), we obtain

$$
|F(x, y)(t)-F(u, v)(t)| \leq M T d(G(x, y), G(u, v)) .
$$

This implies that

$$
d(F(x, y), F(u, v)) \leq \operatorname{MTd}(G(x, y), G(u, v))
$$

for all $x, y, u, v \in X$. Then, hypothesis (h3) is satisfied with $a_{9}=M T<1$ (from condition (f)) and $a_{1}=a_{2}=\ldots=a_{8}=a_{10}=0$.

Now, applying Theorem 2, we obtain the existence of a solution to system (25)(26).

\section{Acknowledgements}

Calogero Vetro was supported by Università degli Studi di Palermo, Local University Project R. S. ex 60\%.

\section{Author details}

${ }^{1}$ Institut Supérieur d'Informatique de Mahdia, Université de Monastir, Route de Rjiche, Km 4, BP 35, Mahdia 5121, Tunisie ${ }^{2}$ Ecole Supérieur des Sciences et Techniques de Tunis, Université de Tunis. 5, Avenue Taha Hussein-Tunis, B. P.:56, Bab Menara-1008, Tunisie ${ }^{3}$ Dipartimento di Matematica e Informatica, Università degli Studi di Palermo, Via Archirafi 34, 90123 Palermo, Italy

\section{Authors' contributions}

All authors contributed equally and significantly in writing this paper. All authors read and approved the final manuscript.

\section{Competing interests}

The authors declare that they have no competing interests.

Received: 5 February 2011 Accepted: 8 August 2011 Published: 8 August 2011

\section{References}

1. Kantorovich, LV: The majorant principle and Newton's method. Dokl Akad Nauk SSSR (NS). 76, 17-20 (1951)

2. Kantorovich, LV: On some further applications of the Newton approximation method. Vestn Leningr Univ Ser Mat Mekh Astron. 12, 68-103 (1957)

3. Vandergraft, JS: Newton's method for convex operators in partially ordered spaces. SIAM J Numer Anal. 4, 406-432 (1967). doi:10.1137/0704037

4. Zabrě̌ko, PP: K-metric and K-normed spaces: survey. Collect Math. 48, 825-859 (1997)

5. Deimling, K: Nonlinear Functional Analysis. Springer (1985)

6. Aliprantis, CD, Tourky, R: Cones and duality. Graduate Studies in Mathematics, American Mathematical Society, Providence, RI. 84 (2007)

7. Huang, LG, Zhang, X: Cone metric spaces and fixed point theorems of contractive mappings. J Math Anal Appl. 332 , 1467-1475 (2007)

8. Abbas, M, Ali Khan, M, Radenović, S: Common coupled fixed point theorems in cone metric spaces for w-compatible mappings. Appl Math Comput. 217, 195-202 (2010). doi:10.1016/j.amc.2010.05.042

9. Altun, I, Damjanović, B, Djorić, D: Fixed point and common fixed point theorems on ordered cone metric spaces. Appl Math Lett. 23, 310-316 (2010). doi:10.1016/j.aml.2009.09.016

10. Beg, I, Azam, A, Arshad, M: Common fixed points for maps on topological vector space valued cone metric spaces. Int J Math Math Sci 2009, 8 (2009). Article ID560264

11. Di Bari, C, Vetro, P: $\varphi$-pairs and common fixed points in cone metric spaces. Rend Cir Mat Palermo. 57, $279-285$ (2008). doi:10.1007/s12215-008-0020-9

12. Di Bari, C, Vetro, P: Weakly $\varphi$-pairs and common fixed points in cone metric spaces. Rend Cir Mat Palermo. 58, 125-132 (2009). doi:10.1007/s12215-009-0012-4

13. Olaleru, JO: Some generalizations of fixed point theorems in cone metric spaces. Fixed Point Theory Appl 2009, 10 (2009). Article ID 657914

14. Rezapour, Sh, Hamlbarani, R: Some notes on the paper "Cone metric spaces and fixed point theorems of contractive mappings". J Math Anal Appl. 345, 719-724 (2008). doi:10.1016/j.jmaa.2008.04.049 
15. Vetro, P: Common fixed points in cone metric spaces. Rend Circ Mat Palermo. 56, 464-468 (2007). doi:10.1007/ BF03032097

16. Bhashkar, TG, Lakshmikantham, V: Fixed point theorems in partially ordered cone metric spaces and applications. Nonlinear Anal. 65, 825-832 (2006). doi:10.1016/j.na.2005.10.015

17. Lakshmikantham, V, Cirić, L: Coupled fixed point theorems for nonlinear contractions in partially ordered metric spaces. Nonlinear Anal. 70, 4341-4349 (2009). doi:10.1016/j.na.2008.09.020

doi:10.1186/1687-1812-2011-27

Cite this article as: Aydi et al: Coupled fixed point results in cone metric spaces for -compatible mappings. Fixed Point Theory and Applications 2011 2011:27.

Submit your manuscript to a SpringerOpen ${ }^{\odot}$ journal and benefit from:

- Convenient online submission

- Rigorous peer review

- Immediate publication on acceptance

- Open access: articles freely available online

- High visibility within the field

- Retaining the copyright to your article

Submit your next manuscript at $\boldsymbol{s p r i n g e r o p e n . c o m ~}$ 